\title{
A Bayesian Non-parametric Mixed-Effects Model of Microbial
}

\section{Phenotypes}

\author{
Peter D. Tonner ${ }^{1,2, \dagger}$, Cynthia L. Darnell ${ }^{2}$, Francesca M.L. Bushell ${ }^{3}$, Peter A. Lund ${ }^{3}$, \\ Amy K. Schmid ${ }^{1,2,4 *}$, Scott C. Schmidler ${ }^{1,5,6}$ \\ ${ }^{1}$ Program in Computational Biology and Bioinformatics, Duke University, Durham, 27708, USA, \\ 2 Biology Department, Duke University, Durham, 27708, USA, \\ ${ }^{3}$ School of Biosciences, University of Birmingham, Birmingham, United Kingdom, \\ ${ }^{4}$ Center for Genomics and Computational Biology, Duke University, Durham, 27708, USA, \\ ${ }^{5}$ Department of Statistical Science, Duke University, Durham, 27708, USA, \\ ${ }^{6}$ Department of Computer Science, Duke University, Durham, 27708, USA, \\ ${ }^{\dagger}$ Current address: National Institute for Standards and Technology, Gaithersburg, MD, 20899, USA, \\ *To whom correspondence should be addressed; E-mail: amy.schmid@duke.edu.
}

\begin{abstract}
Substantive changes in gene expression, metabolism, and the proteome are manifested in overall changes in microbial population growth. Quantifying how microbes grow is therefore fundamental to areas such as genetics, bioengineering, and food safety. Traditional parametric growth curve models capture the population growth behavior through a set of summarizing parameters. However, estimation of these parameters from data is confounded by random effects such as experimental variability, batch effects or differences in experimental material. A systematic statistical method to identify and correct for such confounding effects in population growth data is not currently available. Further, our previous work has demonstrated that parametric models are insufficient to explain and predict microbial response under non-standard growth conditions. Here we develop a hierarchical Bayesian non-parametric model of population growth that identifies the latent growth behavior and response to perturbation, while simultaneously correcting for random effects in the data. This model enables more accurate estimates of the biological effect of interest, while better accounting for the uncertainty due to technical variation. Additionally, modeling hierarchical variation provides estimates of the relative impact of various confounding effects on measured population growth.
\end{abstract}




\section{Introduction}

Population growth phenotypes inform studies in microbiology, including gene functional discovery, bioengineering process development, and food safety testing $1+3$. For example, recent advances in microbial functional genomics and phenotyping, or "phenomics", have enabled transformative insights into gene functions, proving critical for mapping the genotype to phenotype relationship ${ }^{4}$. Methods such as genome-wide CRISPRi5 and targeted genomescale deletion libraries $\sqrt{67}$ frequently rely upon accurate quantitation of microbial population growth as an assay to identify novel mutants with significant growth phenotypes. Population growth is an aggregate measure of all cellular processes and captures how microbial cells adapt and survive in their environmental niche ${ }^{8}$. Because microbial population culturing is a necessary precursor to many experimental procedures in microbiology 9 , reproducible results require accurate quantification of the variability in culture state measured through growth $\frac{910}{10}$.

Typical analyses of microbial population growth involve estimating parametric models under the assumptions of standard growth conditions comprised of three successive growth phases: (1) lag phase, in which the population adapts to a new environment, typically fresh growth medium at culture inoculation; (2) log phase, when the population grows exponentially at a rate dependent on nutrients in the environment; and (3) stationary phase, where measurable population growth terminates thereby reaching the culture carrying capacity 11 . Recent studies have shown that the estimates of parameters in these models are highly uncertain 12 . This uncertainty arises both from factors of biological interest, such as differences in genetic background and environment, as well as uncontrolled technical noise from experimental manipulation of microbial cultures. While such sources of variability can be modeled using fixed and random effects 15 , parametric population growth models have additional limitations. Most notably, when population growth deviates from the standard sigmoidal shape assumed in parametric models, secondary models must be developed on a case by case basis for each new experimental perturbation $20 \mid 21$. Additionally, we have shown in previous work that in cases such as extreme stress or strongly deleterious mutations, no parametric growth model accurately represents the growth curve, regardless of secondary mode $19 \sqrt{1922 \mid 23}$.

Factors affecting microbial growth measurements include both fixed and random effects 24 . Fixed effects are assumed to be drawn from a finite set of perturbations of interest, for example the effect of different concentrations of a chemical on growth that are entirely represented in the dataset. Random effects, conversely, can be viewed as a random sample from a larger population of interest. For example, repeating the same design over many experiments corresponds to sampling the random experimental effect from the theoretical population of all possible experiments that could be conducted with this design $\sqrt{3 \mid 25}$. Random effects arising from repeated experimental design are typically referred to as batch effects ${ }^{26 \mid 27}$. Batch effects are often a significant component of measurement noise in highthroughput genomics experiments 28 . However, random effects are not always due to experimental noise, and may represent quantities of direct scientific interest; for example, assaying a set of genetic backgrounds may be viewed as sampling from the population of all possible genetic variants 29 . Models which include both fixed and random effects are referred to as mixed effects models. 
In this study we present phenom, a general model for analysis of phenomic growth curve experiments based on a Bayesian non-parametric functional mixed effects model of microbial growth. We demonstrate the utility of phenom model to analyze population growth measurements of two microorganisms: the hypersaline adapted archaeon, Halobacterium salinarum; and the opportunistic bacterial pathogen, Pseudomonas aeruginosa. H. salinarum is a model organism for transcriptional regulation of stress response in the third domain of life, the Archaea ${ }^{34} 36$. $H$. salinarum is particularly well adapted to resisting oxidative stress (OS), which arises from the buildup of reactive oxygen species and causes damage to many critical cellular components, including DNA, protein, and lipids3743. Population growth measurements of H. salinarum under OS have been used previously to quantify these harmful effects on physiology, as well as identify regulatory factors important for OS survival 2240,42 . The presence of batch effects in H. salinarum OS response was reported (and corrected for) previously 1 , but did not model individual batch effects for each term in the model. This motivated the explicit deconstruction of batch effects between different factors (e.g. strain and stress), which we have implemented and reported here in phenom.

Pseudomonas aeruginosa is an opportunistic microbial pathogen and a growing problem in hospital-borne infections. Rising antimicrobial resistance of these organisms has necessitated the development of alternative treatment strategies. For example, topical treatment of infected burn wounds with acetic or organic acids (OAs) has been successfu 44 . OA impact on growth depends on external $\mathrm{pH}$ levels — in acidic intracellular environments the OA does not dissociate, freely traverses the cellular membrane as an uncharged particle, and dissociates in the neutral cytoplasm inducing acid stress $\frac{45}{4}$. Here we apply phenom to the $P$. aeruginosa dataset, which is foundational for a larger study of $P$. aeruginosa strains responding to $\mathrm{pH}$ and $\mathrm{OA}$ perturbation as a potential novel treatment of pathogenic bacterial infections 23 .

Stress occurs constantly in the environment: as conditions change, mild to severe cellular damage occurs, and cells must regulate their molecular components to survive 46 49. Population growth measurements are particularly vital to the study of stress response by providing a quantitative measure of growth differences against a non-stressed contro ${ }^{1}$. Our model recovers fixed effects due to high and low levels of oxidative stress in H. salinarum as well as interactions between organic acid concentration and $\mathrm{pH}$ in $P$. aeruginosa, while correcting for random effects from multiple sources, thus enabling more accurate estimates of the significance of the stress treatment effect. Notably, in cases where random effect and fixed effect sizes are comparable, we demonstrate that mixed modeling is critical for accurate quantification of model uncertainty. If random effects are not included in the model, the significance of the effect of stress treatments on population growth can be erroneously overestimated. We discuss the implications of these findings for multiple areas of microbiology research. 


\section{Results}

\subsection{Hierarchical batch effects typical in phenomics datasets render parametric models ineffective}

In the dataset used here, population growth for each of $P$. aeruginosa and $H$. salinarum cultures was monitored under standard (non-stressed) conditions vs. stress conditions (see Materials and Methods and references 22, 23, for precise definition of "standard conditions" for each organism). Specifically, cultures were grown in liquid medium in a high throughput growth plate reader that measured population density at 30 minute intervals over the course of 24 hours (P. aeruginosa) or 48 hours (H. salinarum); the resulting data are shown in Fig. 1. Experimental designs for each organism included biological replicates (growth curves from different colonies on a plate), technical replicates (multiple growth curves from the same colony), varying conditions (stress vs standard), and are further divided into batches (different runs of the high throughput growth plate reader). H. salinarum was grown under high (0.333 mM paraquat (PQ)) and low (0.083 mM PQ) levels of oxidative stress (OS); the data are combined from published $19|22| 41$ and unpublished studies (Fig 1 $\mathrm{A}$ ). The OS responses of $H$. salinarum were compared to a control of standard growth in rich medium, representing optimal conditions for the population. The experimental design was replicated in biological quadruplicate and technical triplicate, across nine batches (Fig. 1A, individual curves and axes). P. aeruginosa was grown in the presence of increasing concentrations of three different organic acid $(\mathrm{OA})$ chemicals $(0-20 \mathrm{mM}$; benzoate, citric acid, and malic acid), each combined with a gradient of $\mathrm{pH}(5.0-$ 7.0) ${ }^{23}$. Each P. aeruginosa growth condition was repeated across 3 biological replicates and two batches (Fig $\left.1 \mathrm{~B}\right)$. The different $P$. aeruginosa and $H$. salinarum experimental designs with varying numbers of replicates at each level provides a rich testbed for exploring the impact of modeling random effects with phenom (Figs. 1 $1 \mathrm{~B}$, , S1, S2).

Figures 1 and 2 demonstrate the two key issues described above and addressed in this paper. First, batch effects are present in both $H$. salinarum and the $P$. aeruginosa datasets. For H. salinarum, clear differences in growth under both standard and stress conditions are observed in the raw data across experimental batches (i.e. separate runs of the growth plate reader instrument; Fig. 11. Some batches show a different phenotype, with either a complete cessation of growth or an intermediate effect with decreased growth relative to standard conditions. For example, in some batches, populations stressed with low OS grow at the same rate and reach the same carrying capacity as populations grown under standard conditions. For $P$. aeruginosa, a clear difference between batches grown under $10 \mathrm{mM}$ citric acid at $\mathrm{pH}=5.5$ is observed [Fig. $1 \mathrm{~B}$ (graph in fourth column, third row) and Fig. 2D]. Like with citric acid, batch effects were also found in some of the other conditions considered (e.g. growth under malic acid, Figs. S1. S2).

Second, standard parametric growth curve models fail to describe experimental measurements adequately (Fig 2A, B), as we have shown previously with both datasets $19|22| 23$. In Fig. 2, we examined the impact of batch and replicate effects on our data by considering how they change parameters estimated from a mixed effects parametric model of population growth ${ }^{32}$. We focused on calculating $\mu_{\max }$, the maximum instantaneous growth rate attained 

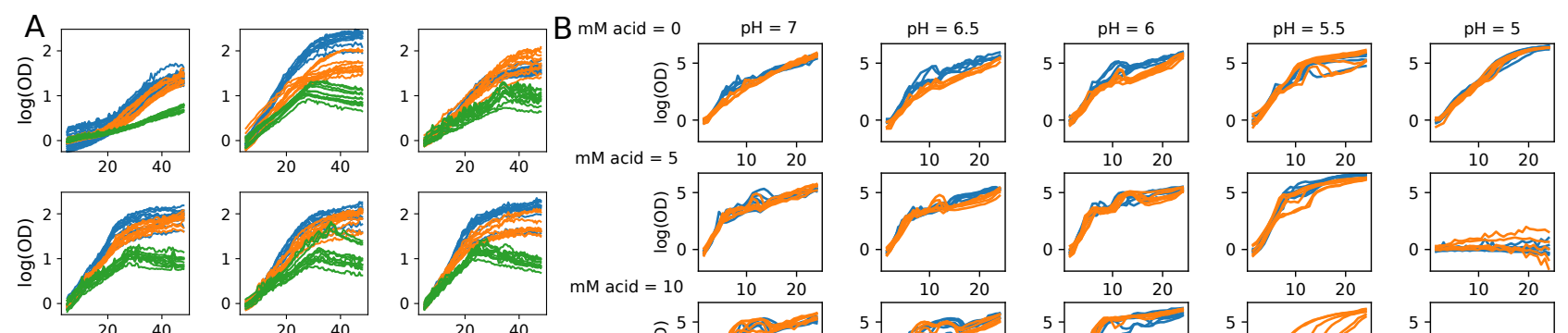

$\mathrm{mM}$ acid $=10 \quad 10 \quad 20$
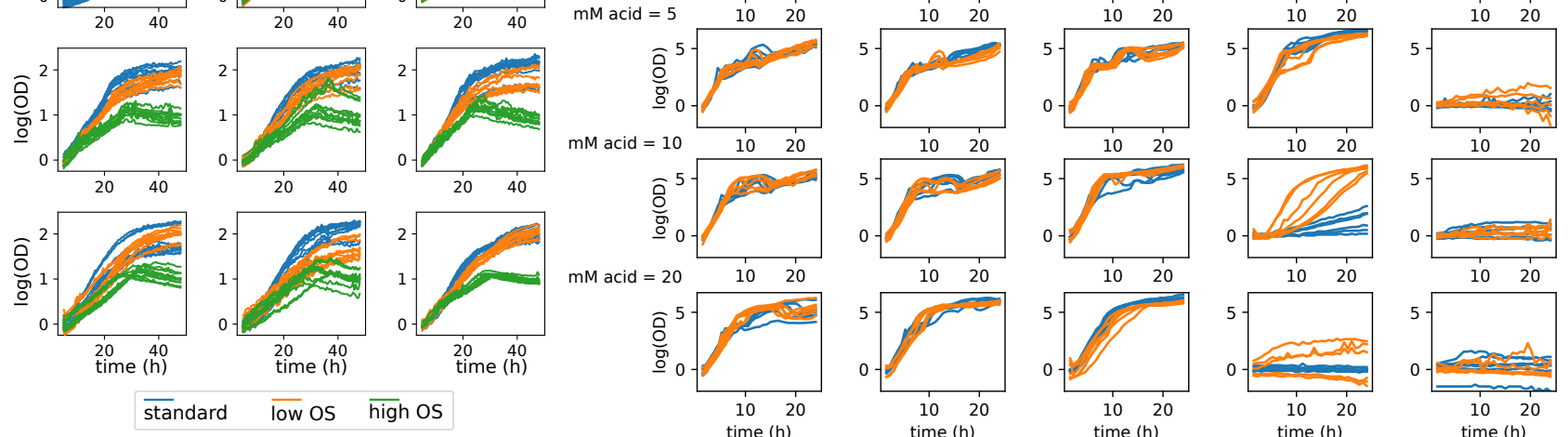

Figure 1: Batch variation in high throughput phenomics studies. (A) Population growth measurements of $H$. salinarum under standard conditions (blue), and low (orange) and high (green) levels of OS. Individual measurement curves are replicates and each graph panel is a different batch. (B) Growth of P. aeruginosa strain PA01 under gradient of $\mathrm{pH}(5-7)$ and citric acid $(0-20 \mathrm{mM})$. Colors represent different batches.

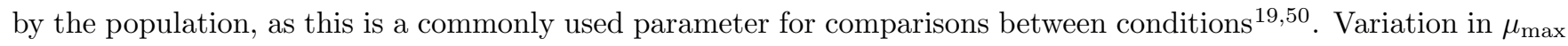
estimates were observed both on the replicate and batch level, as shown by the kernel density estimates (KDE) of $\mu_{\max }$ for each stress level (Fig. S3). The variance in $\mu_{\max }$ is remarkably high: the $95 \%$ confidence interval for $\mu_{\max }$ under standard growth is $0.050-0.141$, a nearly 3-fold change between the lower and upper interval limits. Thus, while the t-test conducted on $\mu_{\max }$ estimates between standard conditions and each stress level is statistically significant (Fig S3), it is difficult to conclude: (a) what the true magnitude of the stress effects may be; and (b) to what degree the variation due to replicate and batch should inform biological conclusions. The error of the logistic growth model under each PQ condition was also examined. Error increased under high OS (Fig S4). High OS induces a growth phenotype that deviates heavily from the sigmoidal growth curve assumed in the logistic model as well as in other commonly used growth models. This leads to a poor fit under the high OS condition as has been shown previously (Fig $\left.\mathrm{S}_{1}^{119}\right)$. The residuals under standard, low, and high OS conditions also appear to be dependent. Our previous work also demonstrated poor fits to the $P$. aeruginosa data using parametric model $\sqrt{23}$. Taken together, the initial assessment of these two datasets indicates that: (a) technical variation due to batch and replicate in growth curve data can be high; and (b) commonly used standard parametric models are not able to adequately capture or correct for these sources of variability. These sources of error need to be corrected in order to model true growth behavior and inform biological conclusions from the data.

\subsection{A hierarchical Bayesian model of functional random effects in microbial growth}

We previously established the ability of non-parametric Bayesian methods to improve the modeling of growth phenotypes $19|22| 23$. Here, we describe phenom, a fully hierarchical Bayesian non-parametric functional mixed effects model for population growth data. We highlight the utility of phenom to correct for confounding, random effects in growth 

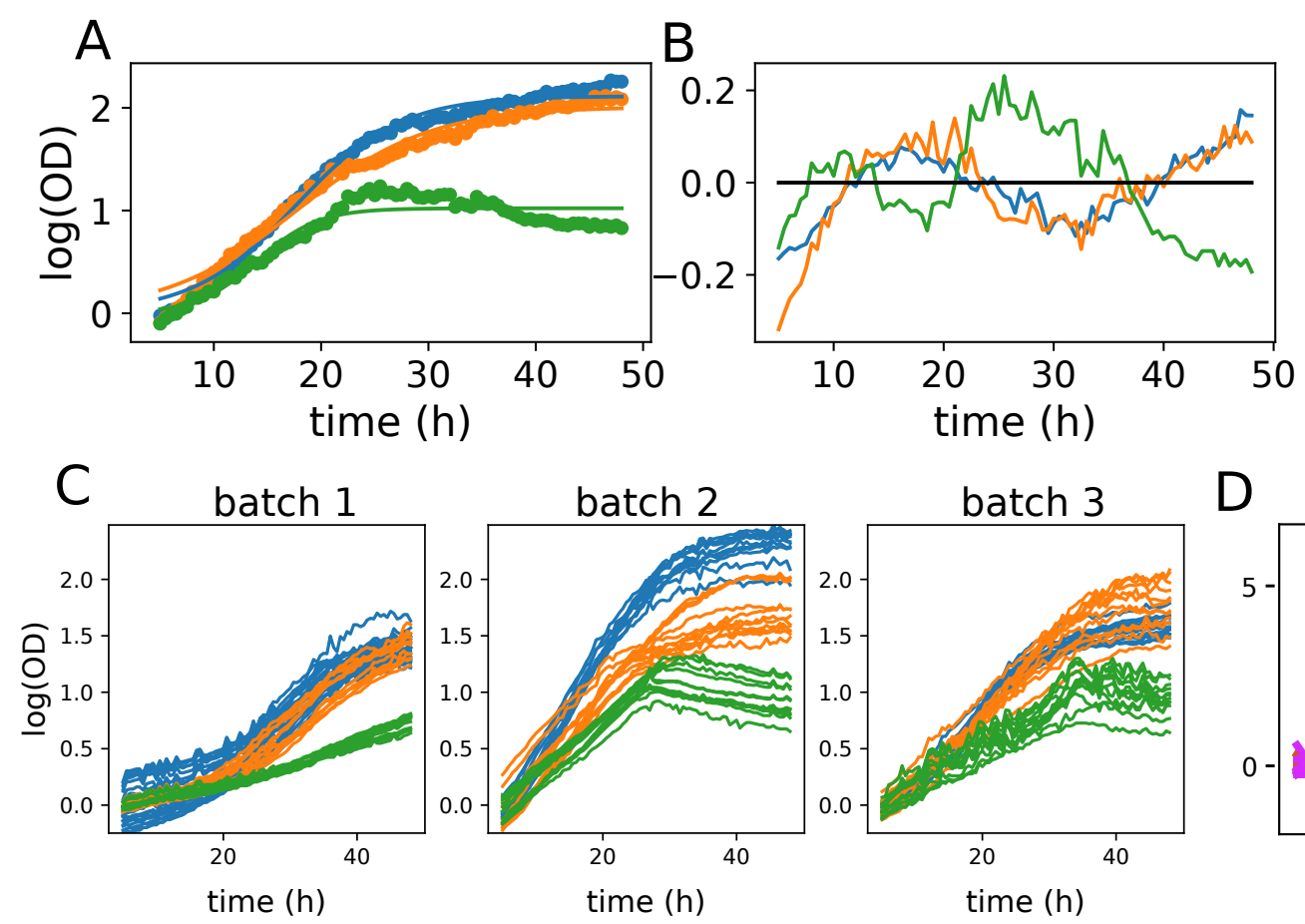

$\mathrm{mM} P Q$

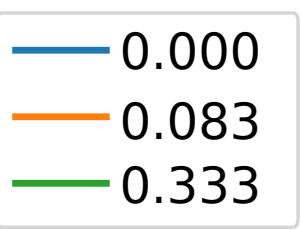

西
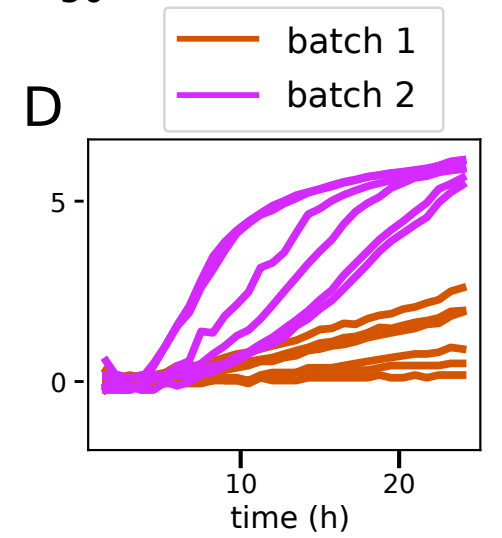

Figure 2: Batch effects are prevalent in microbial phenomic datasets. (A) Parametric fits to H. salinarum growth curves. (B) Residuals of parametric growth curve fit. (C) Growth of H. salinarum under standard conditions (blue), low (orange) and high (green) OS across three batches. (D) Measurement of P. aeruginosa growth under $10 \mathrm{mM}$ citric acid at $5.5 \mathrm{pH}$. Measurements for each condition vary significantly with batch.

phenotypes.

In order to model both biological and technical variation in microbial growth (Fig 3), we first assume that a set of population growth measurements are driven by an (unobserved) population curve $\mu(t)$ (Fig 3 A, blue curve) of unknown shape. For example, $\mu(t)$ might represent the average growth behavior of an organism under standard conditions. This mean growth behavior may be altered by a treatment effect, represented by an additional unknown curve $\delta(t)$ (Fig $3 \mathrm{~A}$, orange curve). For example $\delta(t)$ may represent the effects on growth induced by low or high levels of OS (Fig $2 \mathrm{~A})$. The average growth behavior of a population under stress conditions would then be described by the curve $f(t)=\mu(t)+\delta(t)$.

When considering a combinatorial experimental design, such as that described for $P$. aeruginosa growth (Fig. 1B), we model independent effects of different treatments as well as their interaction via the form:

$$
y(t, i, j)=\mu(t)+\alpha_{i}(t)+\beta_{j}(t)+(\alpha \beta)_{i, j}(t) .
$$

Here, $y(t, i, j)$ denotes the observed population size at time $t$ with treatments $i$ and $j$ of two independent stress conditions. Additionally, $\alpha_{i}(t)$ and $\beta_{j}(t)$ are the independent effects of each stress condition, and $(\alpha \beta)_{i, j}(t)$ is their interaction. This model corresponds to a functional analysis of variance ${ }^{51}$, which we have previously used to estimate independent and interaction effects of microbial genetics and stress 22 . Here, we consider the interaction of the two 


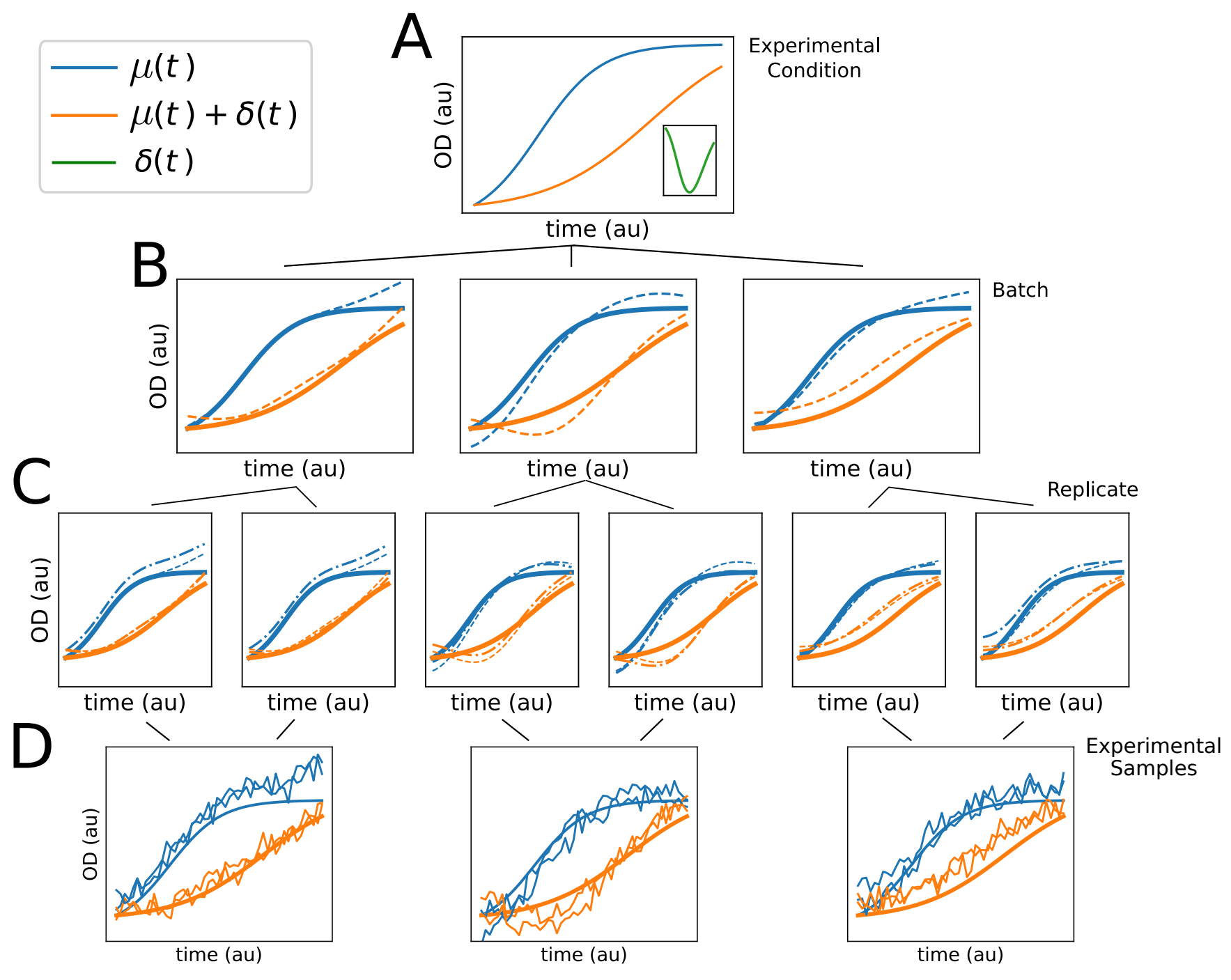

Figure 3: Hierarchical model of functional data. Representative diagram of hierarchical variation present in microbial growth data. Each tier of graphs represents a different variation source, and lines indicate relationship between them: experimental condition is the true growth behavior of interest, with the condition repeated across batches, and replicates repeated within each batch. (A) Functional phenotypes $\mu(t)$ (blue), $\mu(t)+\delta(t)$ (orange), and $\delta(t)$ (green curve in inset). (B) Batch effects on $\mu(t)$ and $\mu(t)+\delta(t)$. Each plot is a different batch, solid lines are the true functions as in (A), and the dashed lines are the observed batch effect of $\mu(t)$ and $\mu(t)+\delta(x)$ for the corresponding batch. (C) Replicate effect within batches. Each axis is a different replicate, solid and dashed lines as in (B), dotted-dashed line is the observed replicate function. (D) Observations from the model described in (A-C). Each curve is sampled with a mean drawn from the global mean, with added batch and replicate effects (dotted-dashed lines in $\mathrm{C}$ ) and iid observation noise. Each axis is a different batch. The smooth solid lines are the true functions $\mu(t)$ and $\mu(t)+\delta(t)$ in (A). 
stress conditions as well as random functional effects in the model.

Variability around these fixed effect growth models is described by additional, random curves associated with two major sources of variation: batch and replicate (Fig $3 \mathrm{~B}, \mathrm{C})$. Batches correspond to a single high-throughput growth experiment and replicates are the individual curve observations within a batch. Using phenom throughout this study, we only compare replicates that are contained within the same batch. This is due to the nested structure between batch and replicates (Fig 3). Noise due to both replicate and batch do not appear to be independent identically distributed (iid), as observed in the correlated residuals around the mean for each experimental variate (Fig. S55A and B). Each observed growth curve is therefore described by a combination of the fixed effects and the corresponding batch and replicate effects (Fig 3D). Both replicate and batch variation are modeled as random effects because the variation due to both sources cannot be replicated, i.e. a specific batch effect cannot be purposefully re-introduced in subsequent experiments. Instead, these variates are assumed to be sampled from a latent super-population $\sqrt{52}$. Combining the fixed and random effects, we arrive at a mixed-effects model of microbial phenotypes.

We adopted a hierarchical Bayesian framework to model these mixed effects. In this framework, batch effects are described by a shared generative distribution, allowing them to take on distinct values while still pooling across replicates for accurately estimating the generating distribution 53 . We use Gaussian process (GP) distributions for all groups in the model. GPs are flexible, non-parametric distributions suitable for smooth functions 54 . To assess the impact of incorporating random effects on estimation of the treatment effect of interest, we analyze three models of increasing complexity: $\mathrm{M}_{\text {null }}$ excludes all hierarchical random effects, $\mathrm{M}_{\mathrm{batch}}$ incorporates batch variation only, and $\mathrm{M}_{\text {full }}$ incorporates both batch and replicate variation. These models, collectively called phenom, were implemented using the probabilistic programming language Stan $\frac{55}{5}$, which efficiently traverses the posterior through Hamiltonian Monte Carlo (see Materials and Methods).

In order to demonstrate the impact of batch effects on the conclusions drawn from the analysis of microbial growth data, we estimated the latent functions driving both $H$. salinarum and $P$. aeruginosa growth using the $\mathrm{M}_{\text {null }}$ model of phenom, with each batch analyzed separately (Fig 4). This corresponds to the analysis that would be conducted after generating any single set of experiments from a batch, without considering or controlling for batch effects, and therefore provides a test of the impact of ignoring batch effects.

For $H$. salinarum, growth data under standard conditions was used to estimate a single mean function, $\mu(t)$ (Fig. 4A). Fixed effects for growth under low and high OS was added as $\delta(t)$ (Fig $4 \mathrm{~B})$. For the P. aeruginosa dataset, batch effects on the interaction between $\mathrm{pH}$ and organic acid concentration was represented by a function $(\alpha \beta)_{p, m}(t)$, again estimated non-parametrically (Fig. $4 \mathrm{C}$ ). However, rather than reporting $(\alpha \beta)_{p, m}(t)$ directly, we report its time derivative, which has the interpretation of instantaneous growth rate rather than absolute amount of growth 5 .

Fitting the $\mathrm{M}_{\text {null }}$ model to each separate batch reveals that the posterior distributions obtained for each function of interest $\left(\mu(t), \delta(t)\right.$, and $\left.(\alpha \beta)_{p, m}(t)\right)$ are highly variable across batches (Fig. 44). This is observed in both the $H$. salinarum and $P$. aeruginosa datasets, where the experimental conditions, and therefore the underlying true functions, remain constant across batches in each case. Such variability can impact conclusions. For example, in the 


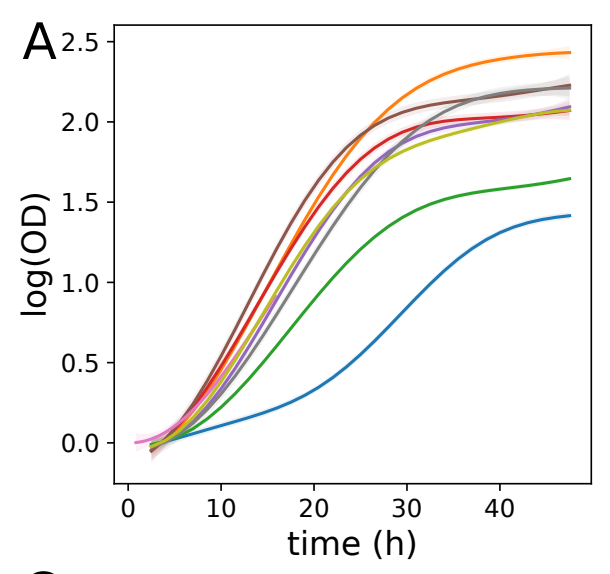

C

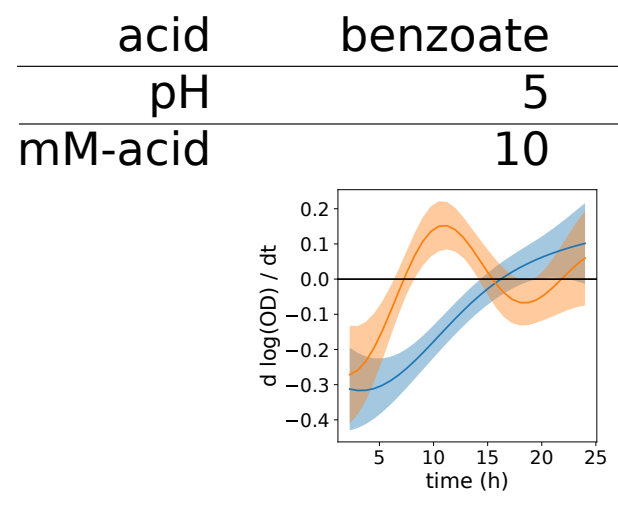

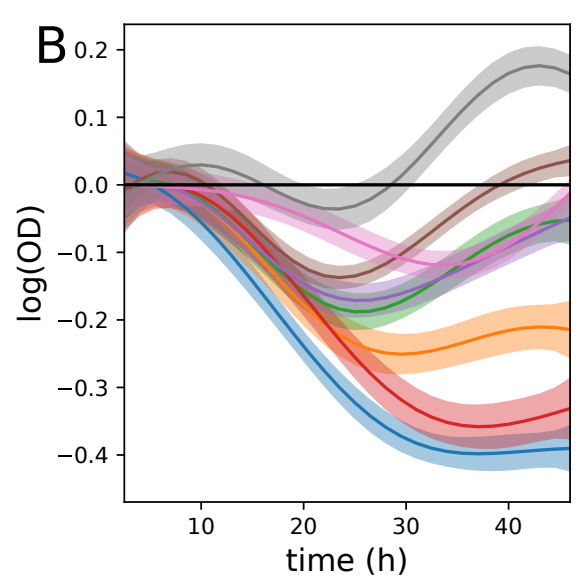

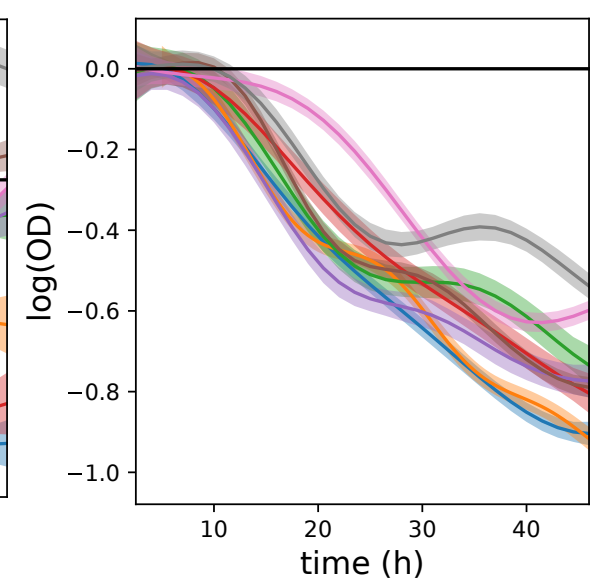

citric

malic

malic

5.5

\section{5} 10
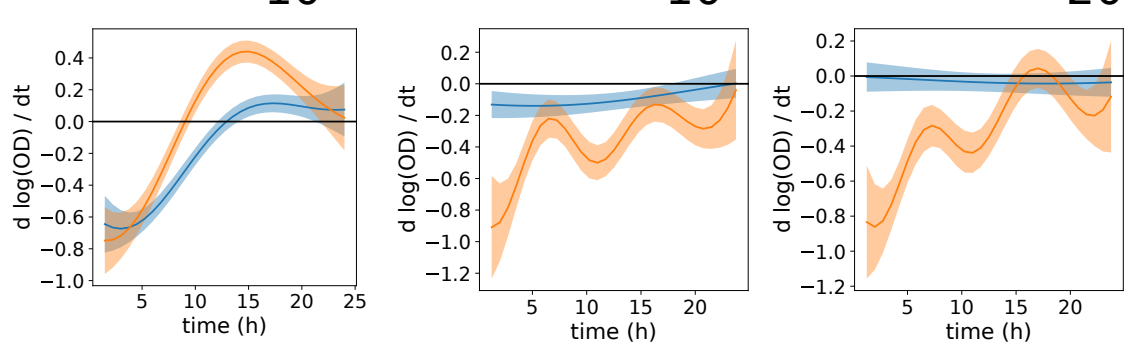

Figure 4: $\mathbf{M}_{\text {null }}$ model estimates are confounded by batch effects. Posterior intervals of functions are shown for different analyses where phenom $\mathrm{M}_{\text {null }}$ was fit using data from each batch separately. In all plots, solid line represents posterior mean, shaded region indicates $95 \%$ credible region, and each color corresponds to a different posterior conditioned on data from a single batch. (A) Posterior intervals of $\mu(x)$, the standard growth phenotype of H. salinarum. (B) Posterior interval of $\delta(x)$ under low (left) and high (right) OS response of H. salinarum. (C) Posterior interval of interaction function $(\alpha \beta)_{p, m}(t)$ for $P$. aeruginosa growth in indicated $\mathrm{pH}$ and acid concentration. 
low OS condition in the $H$. salinarum dataset, both the statistical significance of $\delta(t)$ and the sign (improved vs. impaired growth) differs between batches (Fig $4 \mathrm{~B}$, left). A similar batch variability was observed under high OS, but due to the stronger effect of the stress perturbation, estimates of $\delta(t)$ are less affected by batch and replicate variation (Fig $4 \mathrm{~B}$, right). Similarly, the batch variability observed in the raw $P$. aeruginosa growth data (Fig. $1 \mathrm{~B}$ ) results in significantly different posterior estimates of the interaction effect $(\alpha \beta)_{p, m}(t)$ across batches (Fig. 4 C). Differences observed include the timing and length of negative growth impact (benzoate and citric acid), and completely opposite effects with either strong or no interaction (malic acid). In addition, the posterior variance of each function, which indicates the level of uncertainty remaining, is low for each batch modeled separately. This indicates high confidence in the estimated function despite observed differences across batches. These analyses suggest that use of a single experimental batch leads to overconfidence in explaining the true underlying growth behavior.

\subsection{Hierarchical models correct for batch effects in growth data}

To demonstrate the use of phenom to combat the impact of batch effects on growth curve analysis, we combined data across all batches and performed the analysis using each of the $\mathrm{M}_{\text {null }}, \mathrm{M}_{\text {batch }}$, and $\mathrm{M}_{\text {full }}$ models (Fig. 5). Estimates of $\mu(t)$ between each model were largely similar, likely due to the abundance of data present to estimate this variable (Fig. S6). Instead, we focus on the estimates of $\delta(t)$ for low and high OS response of $H$. salinarum (Fig $5 \mathrm{~A}$ ) and the interaction $(\alpha \beta)_{p, m}$ between $\mathrm{pH}$ and $\mathrm{OA}$ concentration effects on P. aeruginosa growth (Fig. $\left.5 \mathrm{C}\right)$.

Growth impairment in the presence of low OS relative to standard conditions (i.e. $\delta(t)$ ) is estimated to be significant during the time points of $\sim 10-40$ hours under $\mathrm{M}_{\text {null }}$. In contrast, only time points $\sim 20-40$ are significantly non-zero under $\mathrm{M}_{\text {batch }}$ (Fig. 5A, left). Although $\mathrm{M}_{\text {full }}$ and $\mathrm{M}_{\text {null }}$ exhibit similar regions of time where effects are significant, uncertainty is higher (confidence bands wider) when batch and replicate effects are taken into account $\left(\mathrm{M}_{\text {full }}\right)$. Given the stronger stress effect in the high OS condition (Fig. 5A, right), estimates of $\delta(t)$ were significantly non-zero under all three models, with only minor differences between the three model estimates. Importantly, we note that the posterior interval of $\delta(t)$ under $\mathrm{M}_{\text {null }}$ for low OS does not include the best approximation of the true function (the posterior mean of $\delta(t)$ under $\mathrm{M}_{\text {full }}$ ) for greater than $80 \%$ of the time course (Fig. 5B). Taken together, these results suggest that certain time points where $\delta(t)$ is concluded to be non-zero under $\mathrm{M}_{\text {null }}$ may be inaccurate, especially for stress conditions with modest effects on growth phenotype.

The impact of modeling hierarchical variation on estimating interaction effects in $P$. aeruginosa growth was condition dependent (Fig. 5C). Across conditions, however, a decrease in posterior certainty on the true shape of the underlying function was again observed under $\mathrm{M}_{\text {batch }}$ and $\mathrm{M}_{\text {full }}$. For example, the interaction between benzoate and $\mathrm{pH}$ became less pronounced under $\mathrm{M}_{\text {full }}$. Similarly, the models of $(\alpha \beta)_{p, m}(t)$ under citric and malic acid showed shrinkage toward zero under $\mathrm{M}_{\text {batch }}$ and $\mathrm{M}_{\text {full }}$. Such shrinkage is a common observance in hierarchical modeling $\sqrt{53}$. Taken together, these results for $P$. aeruginosa extend those previously published ${ }^{23}$, which only included analysis using the $\mathrm{M}_{\text {null }}$ model.

For both $H$. salinarum response to OS and $P$. aeruginosa growth under $\mathrm{pH}$ and $\mathrm{OA}$ exposure, an increase in 

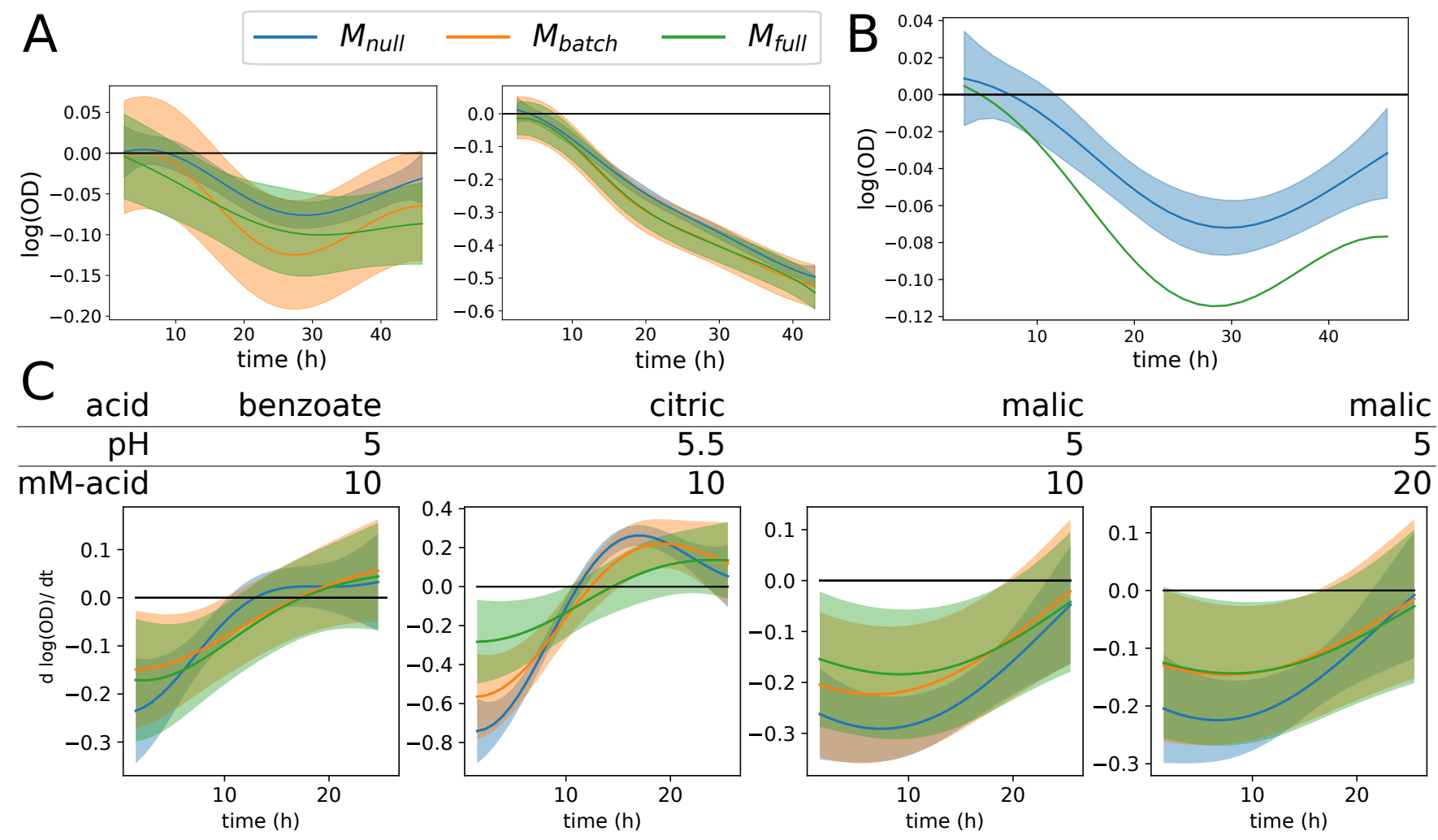

Figure 5: Hierarchical models of growth control for batch effects. Posterior intervals of functions estimated by models of increasing hierarchical complexity: $\mathrm{M}_{\text {null }}$ (blue), $\mathrm{M}_{\text {batch }}$ (orange), and $\mathrm{M}_{\text {full }}$ (green). Solid line indicates posterior mean and shaded regions indicate $95 \%$ credible regions. (A) Posterior interval of $\delta(x)$ for low (left) and high (right) OS response by H. salinarum. (B) Posterior interval of $\delta(x)$ under $\mathrm{M}_{\text {null }}$ (blue shaded region) compared to the posterior mean of $\mathrm{M}_{\text {full }}$ (green line). (C) Posterior interval of interaction function $(\alpha \beta)_{p, m}(t)$ for P. aeruginosa growth in indicated $\mathrm{pH}$ and organic acid concentration. 
posterior variance was observed under $\mathrm{M}_{\text {batch }}$ and $\mathrm{M}_{\text {full }}$ compared to $\mathrm{M}_{\text {null }}$ (Fig S7). However, posterior variance of $\delta(t)$ in the $H$. salinarum OS response was higher under $\mathrm{M}_{\text {batch }}$ compared to $\mathrm{M}_{\text {full }}$. In this case, controlling for replicate effects appears to increase the signal needed to identify $\delta(t)$. In contrast, these variances are equal in the P. aeruginosa data, indicating that the relative improvement in variance afforded by modeling batch vs. replicate effects may be dataset dependent.

\subsection{Variance components demonstrate the importance of controlling for batch effects.}

Variance components, which correspond to the estimated variance of each effect in the model, can be used to compare the impact each group has on the process of interest ${ }^{24}$. To better understand sources of variability in growth curve studies, we used phenom to estimate the variance components for each dataset above. In our hierarchical nonparametric setup, these variance components are the variance hyperparameters (e.g. $\sigma^{2}$ ) of the Gaussian process kernels for each fixed and random effect group. These parameters control the magnitude of function fluctuations modeled by the GP distribution. Larger variance implies higher effect sizes and therefore a larger impact on the observations.

We show the value of variance components by considering the effects identified by $\mathrm{M}_{\text {full }}$ for $H$. salinarum under low OS (Fig. 6). The variance of the data is partitioned between the mean growth $(\mu(t))$, the OS $(\delta(t))$, batch effects (batch curves of $\mu(t)$ and $\delta(t)$ ), biological noise (e.g. replicate variability) and instrument noise $\left(\sigma_{y}^{2}\right)$. This analysis confirms that batch effects, compared to the other sources of experimental variability in the dataset (replicate noise and measurement error), are between 2 to 10 times more impactful on the phenotype measurements. Additionally, variance components enable comparisons between the experimental and treatment factors in the data. Of particular note is that the variance of the treatment of interest, $\delta(t)$, and the batch effects are similar in magnitude, at least in the case of a low-magnitude stress such as $0.083 \mathrm{PQ}$ for $H$. salinarum. This suggests that proper modeling of this treatment requires both sufficient batch replication and accurate modeling of batch effects in those data. Future studies of similar phenotypes can be guided by these estimates in experimental design, choosing an appropriate batch replication for the degree of noise expected 57 . However, the extent of replication required may depend upon the dataset (factorial design, treatment severity, etc). Taken together, variance components provide an aggregated view of the contribution by various factors and guide future experimentation.

\section{Discussion}

We have provided a framework to test and control for random effects in microbial growth data using the hierarchical non-parametric Bayesian model, phenom (Fig. 3). Analysis with phenom indicates that random effects (both batch and replicate) appear in the two microbial population growth datasets studied here, and constitute significant portions of the variability (Fig. 1). Failure to correct for these effects confounds the interpretation of growth phenotypes for factors of interest in a large scale phenotyping analysis (Fig. 4). phenom controls for these random effects and 


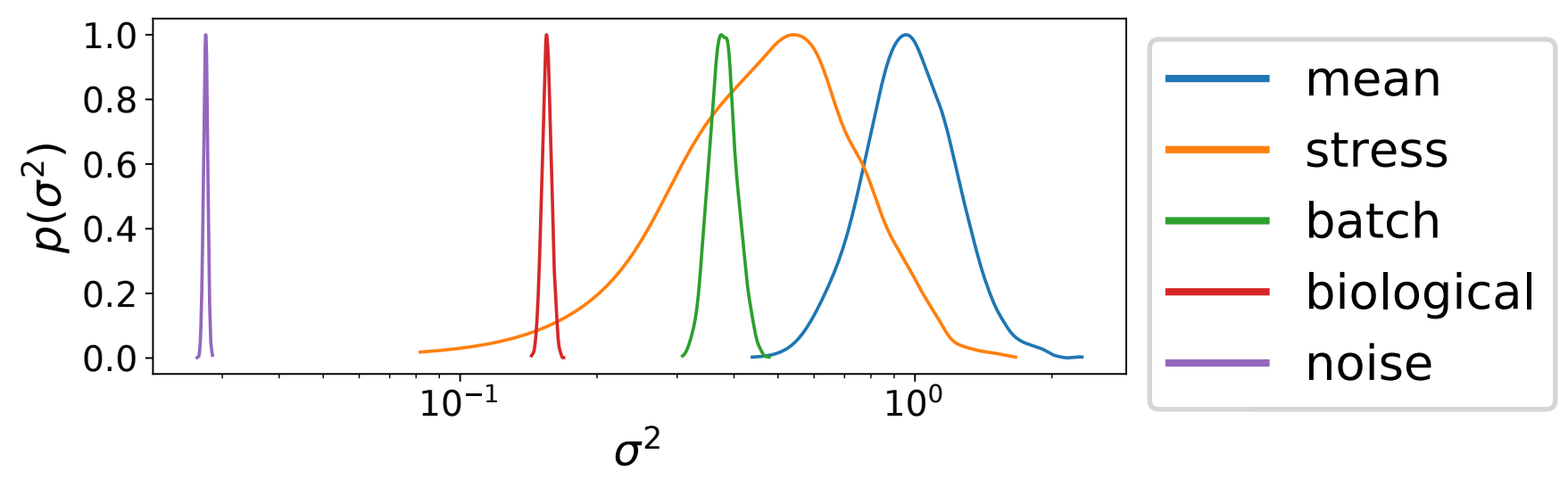

Figure 6: Posterior variance components in the phenom hierarchical phenotype model. Posterior intervals are shown for the kernel variance hyperparameter for different groups of effects from phenom estimated on $H$. salinarum growth under low OS. Groups correspond to $\mu(t)$ (mean), $\delta(t)$ (stress), batch effects (batch), replicate noise (biological), and measurement error (noise).

provides accurate estimates of the growth behavior of interest (Fig. 5). Additionally, phenom can be used to estimate variance components, providing information about the relative impact of various sources of noise in the data (Fig. 6). Controlling for batch effects in these datasets was therefore key to making accurate biological conclusions.

Related fields of functional genomics, such as transcriptomics, have seen considerable interest in controlling for different experimental sources of variation, broadly labeled as batch effect: $28[57 / 62$. These studies have shown that differences between batches first need to be corrected to avoid erroneous conclusions 53 . Here we have shown that, like in transcriptomics data, controlling for sources of variation in phenomics data - particularly due to batch - are an important step in making accurate biological conclusions regarding population growth.

phenom establishes a complete and general method of controlling batch effects in microbial growth phenotypes, overcoming significant weaknesses of previously developed techniques. In reference [19] we identified and corrected for batch effects in a single transcription factor mutant's stress response, but this model did not provide an explicit deconstruction of batch effects between different factors (e.g. strain and stress) and could therefore not determine which factors were most strongly impacted by batch effects. Moreover, this approach utilized a standard GP regression framework, but the standard framework has well-established limitations on dataset size, limiting its applicability to the large datasets we consider here. In reference 22 we described a functional ANOVA model for microbial growth phenotypes, which corresponds to the $\mathrm{M}_{\text {null }}$ model in the phenom case. Again, a global batch effects term was included but individual batch effects were not modeled, and the computational approach utilized (Gibbs sampling) was prohibitively slow for the complete phenom model.

Although we focus here on replicate and batch variation, the phenom model is easily extended to incorporate alternative or additional random and fixed effects appropriate for settings with other sources of variation. For example, depending on the experimental design, phenom could control for variation among labs, experimental material, culture history, or genetic background $\sqrt{25 / 64}[70$ phenom flexibly incorporates additional sources of variation and/or 
interaction between design variables, as demonstrated with the two different designs analyzed for $H$. salinarum and P. aeruginosa here. This flexibility allows phenom to be applied to control for many sources of technical variation within microbial population growth data, thereby improving the analysis and resulting conclusions regarding quantitative microbial phenotypes.

\section{Materials and Methods}

\subsection{Experimental Growth Data}

H. salinarum growth was performed as described previously 22 . Briefly, starter cultures of $H$. salinarum NRC-1 $\Delta$ ura 3 control strain 171 were grown at $42^{\circ} \mathrm{C}$ with shaking at 225 r.p.m. to an optical density at $600 \mathrm{~nm}\left(\mathrm{OD}_{600}\right)$ $\sim 1.8-2.0$ in $3 \mathrm{~mL}$ of Complete Medium $(\mathrm{CM} ; 250 \mathrm{NaCl}, 20 \mathrm{~g} / \mathrm{l} \mathrm{MgSO} 4 \bullet 7 \mathrm{H} 2 \mathrm{O}, 3 \mathrm{~g} / 1$ sodium citrate, $2 \mathrm{~g} / \mathrm{l} \mathrm{KCl,} 10$ $\mathrm{g} / \mathrm{l}$ peptone) supplemented with uracil $(50 \mu \mathrm{g} / \mathrm{ml})$. Cultures were then diluted to $\mathrm{OD}_{600} \sim 0.05$ in a high throughput microplate reader (Bioscreen C, Growth Curves USA, Piscataway, NJ), and growth was monitored automatically by $\mathrm{OD}_{600}$ every 30 minutes for 48 hours at $42^{\circ} \mathrm{C}$. High and low levels of OS were induced by adding $0.333 \mathrm{mM}$ and $0.083 \mathrm{mM}$ of paraquat to the media, respectively, at culture inoculation.

For P. aeruginosa, laboratory strain PAO1 (ATCC 15692) was grown as described in reference 23. Briefly, cultures were grown in M9 minimal media supplemented with $0.4 \%(\mathrm{w} / \mathrm{v})$ glucose and $0.2 \%(\mathrm{w} / \mathrm{v})$ casamino acids and buffered with $100 \mathrm{mM}$ each of MES and MOPS buffers. Population growth was measured with a CLARIOstar automated microplate reader (BMG Labtech) at $37^{\circ} \mathrm{C}$ with $300 \mathrm{rpm}$ continuous shaking. The $\mathrm{OD}_{600}$ was recorded automatically every 15 minutes for a total of 24 hours. A full factorial design of $\mathrm{pH}$ and OA concentration was performed for benzoate, citric acid, and malic acid. An experimental batch corresponded to two repetitions of the experiment on separate days with a minimum of three biological replicates of each condition on each day. Two batches for each OA were performed.

All data generated or analysed during this study are included in this published article (see supplementary information files).

\subsection{Parametric growth curve estimation}

For comparison with our non-parametric methods, parametric growth curve models were estimated using the grofit package in $\mathrm{R}$ with default parameters ${ }^{72}$. The logistic model was used to fit each curve. Kernel density estimates of parameter distributions were calculated with the scipy package with default kernel bandwidth parameters ${ }^{73}$. 


\section{3 phenom: a hierarchical Gaussian process model of microbial growth}

\subsubsection{Gaussian Processes}

A Gaussian process (GP) defines a non-parametric distribution over functions $f(t)$, defined by the property that any finite set of observations of $f$ follow a multivariate normal distribution $\frac{\sqrt{54}}{2}$. A GP is fully defined by a mean function $m(t)$ and a covariance function $\kappa\left(t, t^{\prime}\right)$ :

$$
f(t) \sim G P\left(m(t), \kappa\left(t, t^{\prime}\right)\right) .
$$

GPs are commonly used for non-parametric curve fitting $\sqrt{54}$ where $m(t)$ is typically set to 0 , which we do here. Similarly, we use a common choice for covariance function defined by a radial basis function (RBF) kernel:

$$
\kappa\left(t, t^{\prime}\right)=\sigma^{2} \cdot \exp \left(\frac{-\left|t-t^{\prime}\right|^{2}}{\ell}\right)
$$

where $\sigma^{2}$ is the variance and $\ell$ is the length-scale. The parameter $\sigma^{2}$ controls the overall magnitude of fluctuation in the population of functions described in the GP distribution, while $\ell$ controls the expected smoothness, with larger $\ell$ making smoother, slower varying functions more likely. In the process of non-parametric modeling of growth curves, these parameters are adaptively estimated from the dataset.

\subsubsection{Fixed effects}

We first define the fixed effects models used in this study; these will be augmented with random effects in the next section. We consider fixed effects models of increasing complexity: a mean growth phenotype, a single treatment phenotype, and a combinatorial phenotype with interactions between treatments. All of these models fall under the functional analysis of variance (ANOVA) framework ${ }^{22174}$. To estimate a mean growth profile, as in the case of measuring a single condition, a mean function $\mu(t)$ is estimated from the data by modeling each replicate $y_{r}(t)$ for $1 \leq r \leq R$ as consisting of an unknown mean function observed with additive noise:

$$
y_{r}(t)=\mu(t)+\epsilon_{r}(t),
$$

where $\mu(t) \sim G P\left(0, \kappa_{\mu}\left(t, t^{\prime}\right)\right)$ provides a prior distribution over $\mu$, and $\kappa_{\mu}$ is an RBF kernel with hyperparameters $\left\{\sigma_{\mu}^{2}, \ell_{\mu}\right\}$. Here $\epsilon_{r}(t) \sim N\left(0, \sigma_{y}^{2} I\right)$ is Gaussian white noise.

When estimating the effect of a perturbation on growth, as in the case of OS, we add a second function $\delta(t)$ that represents the effect of the stress being considered. The model then becomes

$$
y_{r}(t)= \begin{cases}\mu(t)+\epsilon_{r}(t) & \text { if standard growth } \\ \mu(t)+\delta(t)+\epsilon_{r}(t) & \text { otherwise }\end{cases}
$$


where $\delta(t) \sim G P\left(0, \kappa_{\delta}\left(t, t^{\prime}\right)\right)$ also follows a GP prior independently of $\mu$, and $\kappa_{\delta}$ has hyperparameters $\left\{\sigma_{\delta}^{2}, \ell_{\delta}\right\}$.

When incorporating possible interaction effects such as those between $\mathrm{pH}$ and organic acids in the $P$. aeruginosa dataset, the model becomes

$$
y_{r}(t, p, m)= \begin{cases}\mu(t)+\epsilon_{r}(t), & \text { if } p=7 \text { and } m=0 \\ \mu(t)+\alpha_{p}(t)+\epsilon_{r}(t), & \text { if } p \neq 7 \text { and } m=0 \\ \mu(t)+\beta_{m}(t)+\epsilon_{r}(t), & \text { if } p=7 \text { and } m \neq 0 \\ \mu(t)+\alpha_{p}(t)+\beta_{m}(t)+(\alpha \beta)_{p, m}(t)+\epsilon_{r}(t), & \text { otherwise, }\end{cases}
$$

for $\mathrm{pH} p$ and molar acid concentration $m$, with $\alpha_{p}(t)$ representing the main effect of $\mathrm{pH}, \beta_{m}(t)$ the main effect of acid concentration, and $(\alpha \beta)_{p, m}(t)$ the interaction between them. Each effect is drawn from a treatment specific GP prior:

$$
\begin{aligned}
\alpha_{p}(t) & \sim G P\left(0, \kappa_{\alpha}\left(t, t^{\prime}\right)\right) \\
\beta_{m}(t) & \sim G P\left(0, \kappa_{\beta}\left(t, t^{\prime}\right)\right) \\
(\alpha \beta)_{p, m}(t) & \sim G P\left(0, \kappa_{\alpha \beta}\left(t, t^{\prime}\right)\right) .
\end{aligned}
$$

Again, each covariance function is specified by a RBF kernel with corresponding variance and lengthscale hyperparameters that adapt to the observed data. All models in this section correspond to $\mathrm{M}_{\text {null }}$ for their respective analyses, as they do not include any random effects.

\subsubsection{Random effects}

The first random effects added to the model were those used to account for batch effects, in the model $\mathrm{M}_{\mathrm{batch}}$. Under this model, each fixed functional effect becomes the mean of a GP describing the population of possible batch-specific mean curves. For example, under the model of mean growth behavior (Eq. 4), replicate $r$ from batch $i$ is modeled as

$$
y_{i, r}(t)=\mu_{i}(t)+\epsilon_{i, r}(t),
$$

where $\mu_{i}$ is the batch mean drawn from $\mu_{i}(t) \sim G P\left(\mu(t), \kappa_{\mu \text {,batch }}\left(t, t^{\prime}\right)\right)$ with kernel $\kappa_{\mu, \text { batch }}$ and $\epsilon_{i, r}(t) \sim N\left(0, \sigma_{y}^{2} I\right)$. Other $\mathrm{M}_{\text {null }}$ models are converted to $\mathrm{M}_{\mathrm{batch}}$ similarly, with each fixed effect becoming a mean of a GP prior for each batch effect. $\mathrm{M}_{\text {full }}$ develops the hierarchy one step deeper by adding replicate effects to $\mathrm{M}_{\text {batch }}$. Specifically, the error model $\epsilon_{i, r}$ is now described by a GP: $\epsilon_{i, r} \sim G P\left(0, \kappa_{y}\left(t, t^{\prime}\right)\right)$ with corresponding hyperparameters, accounting for replicate-specific variability rather than simply white noise. 


\subsubsection{Inference}

As noted above, each group GP prior is specified by its own RBF kernel with corresponding variance and length-scale parameters $\left(\theta_{l}=\left\{\sigma_{l}^{2}, \ell_{l}\right\}\right)$. For each group, $\sigma_{l}^{2}$ is assigned a $\operatorname{Gamma}(\alpha, \beta)$ prior and $\ell_{l}$ a conjugate inverse-Gamma prior, with user-defined hyperparameters. Noise variance $\sigma_{y}^{2}$ was also assigned a gamma prior. Bayesian inference was then performed, with the posterior distribution obtained by sampling using Markov chain Monte Carlo (MCMC) implemented with the Stan library, which uses a Hamilitonian Monte-Carlo procedure with No-U-turn sampling $\sqrt{55}$. Multiple chains were run to diagnose convergence, with all parameter posterior means confirmed to have converged within $\hat{R}<1.1$ as recommended ${ }^{75}$.

\subsection{Data and Code Availability}

All code for this study is available at https://github.com/ptonner/phenom Raw growth data are available for $H$. salinarum in reference 22 and at https://github.com/ptonner/hsalinarum_tf_phenotype. Raw growth data are available for $P$. aeruginosa in reference 23 and at https://github.com/amyschmid/pseudomonas-organic-acids.

\section{Acknowledgements}

\section{$5.1 \quad$ Funding}

This study was funded by National Science foundation grants DMS-1407622 to SCS; NSF-MCB-1615685, -1651117, and -1642283 to AKS; NSF Graduate Research Fellowship to PDT. Research on this project in PAL's laboratory was supported by grant number BB/K019171/1 from the UK Biotechnology and Biological Sciences Research Council.

\section{$5.2 \quad$ Author Contributions}

PT, AS, PL, and SS conceived of the study. AS, SS, PL directed and provided oversight, training, and funding for the study. PT performed analysis and generated software. CD and FB performed experiments. PT, AS, and SS wrote the manuscript. All authors contributed to the final draft.

\section{References}

1. Darnell, C. L. \& Schmid, A. K. Systems biology approaches to defining transcription regulatory networks in halophilic archaea. Methods. Bacterial and Archaeal Transcription 86, 102-114 (Sept. 2015).

2. Woodruff, L. B. A., Boyle, N. R. \& Gill, R. T. Engineering improved ethanol production in Escherichia coli with a genome-wide approach. Metabolic Engineering 17, 1-11 (May 2013). 
3. Aryani, D. C., den Besten, H. M. W., Hazeleger, W. C. \& Zwietering, M. H. Quantifying strain variability in modeling growth of Listeria monocytogenes. International Journal of Food Microbiology 208, 19-29 (Sept. 2015).

4. Gray, A. N. et al. High-throughput bacterial functional genomics in the sequencing era. Curr. Opin. Microbiol. 27, 86-95 (2015).

5. Peters, J. M. et al. A Comprehensive, CRISPR-based Functional Analysis of Essential Genes in Bacteria. Cell 165, 1493-1506 (2016).

6. Koo, B. M. et al. Construction and Analysis of Two Genome-Scale Deletion Libraries for Bacillus subtilis. Cell Syst 4, 291-305 (2017).

7. Baba, T. et al. Construction of Escherichia coli K-12 in-frame, single-gene knockout mutants: the Keio collection. Mol. Syst. Biol. 2, 2006.0008 (2006).

8. Schaechter, M. From growth physiology to systems biology. International Microbiology: The Official Journal of the Spanish Society for Microbiology 9, 157-161 (Sept. 2006).

9. Egli, T. Microbial growth and physiology: a call for better craftsmanship. Frontiers in Microbiology 6 (Apr. 2015).

10. Neidhardt, F. C. Apples, oranges and unknown fruit. Nature Reviews Microbiology 4, 876-876 (Dec. 2006).

11. Zwietering, M. H., Jongenburger, I., Rombouts, F. M. \& van 't Riet, K. Modeling of the Bacterial Growth Curve. Applied and Environmental Microbiology 56, 1875-1881 (June 1990).

12. Pouillot, R., Albert, I., Cornu, M. \& Denis, J.-B. Estimation of uncertainty and variability in bacterial growth using Bayesian inference. Application to Listeria monocytogenes. International Journal of Food Microbiology 81, 87-104 (Mar. 2003).

13. Nauta, M. J. Separation of uncertainty and variability in quantitative microbial risk assessment models. International Journal of Food Microbiology 57, 9-18 (June 2000).

14. Aryani, D. C., Besten, H. M. W. d. \& Zwietering, M. H. Quantifying Variability in Growth and Thermal Inactivation Kinetics of Lactobacillus plantarum. Applied and Environmental Microbiology 82, 4896-4908 (Aug. 2016).

15. Lianou, A. \& Koutsoumanis, K. P. Strain variability of the behavior of foodborne bacterial pathogens: A review. International Journal of Food Microbiology 167, 310-321 (Nov. 2013).

16. Arroyo-Lpez, F. N., Orli, S., Querol, A. \& Barrio, E. Effects of temperature, pH and sugar concentration on the growth parameters of Saccharomyces cerevisiae, S. kudriavzevii and their interspecific hybrid. International journal of food microbiology 131, 120-127 (May 2009). 
17. Liu, B., Liu, H., Pan, Y., Xie, J. \& Zhao, Y. Comparison of the Effects of Environmental Parameters on the Growth Variability of Vibrio parahaemolyticus Coupled with Strain Sources and Genotypes Analyses. Frontiers in Microbiology 7 (June 2016).

18. Carlin, F. et al. Variation of cardinal growth parameters and growth limits according to phylogenetic affiliation in the Bacillus cereus Group. Consequences for risk assessment. Food Microbiology 33, 69-76 (Feb. 2013).

19. Tonner, P. D., Darnell, C. L., Engelhardt, B. E. \& Schmid, A. K. Detecting differential growth of microbial populations with Gaussian process regression. Genome Research 27, 320-333 (Nov. 2017).

20. Peleg, M \& Corradini, M. Microbial growth curves: what the models tell us and what they cannot. Critical Reviews in Food Science and Nutrition 51, 917-945 (Dec. 2011).

21. Peleg, M., Normand, M. D. \& Corradini, M. G. The Arrhenius Equation Revisited. Critical Reviews in Food Science and Nutrition 52, 830-851 (Sept. 2012).

22. Darnell, C. L., Tonner, P. D., Schmidler, S. \& Schmid, A. K. Systematic discovery of archaeal transcription factor functions in regulatory networks through quantitative phenotyping analysis. mSystems 2, e00032-17 (2017).

23. Bushell, F. M. L., Tonner, P. D., Jabbari, S., Schmid, A. K. \& Lund, P. A. Synergistic Impacts of Organic Acids and $\mathrm{pH}$ on Growth of Pseudomonas aeruginosa: A Comparison of Parametric and Bayesian Non-parametric Methods to Model Growth. Frontiers in Microbiology 9, 3196 (2019).

24. Searle, S., Casella, G. \& McCulloch, C. Variance Components (Wiley, 1992).

25. Van Derlinden, E., Lule, I., Boons, K. \& Van Impe, J. On the influence of the experimental set-up on the heterogeneous heat response of E. coli K12. Procedia Food Science. 11th International Congress on Engineering and Food (ICEF11) 1, 1067-1072 (Jan. 2011).

26. Churchill, G. A. Fundamentals of experimental design for cDNA microarrays. Nature Genetics 32, 490-495 (Dec. 2002).

27. Baryshnikova, A. et al. Quantitative analysis of fitness and genetic interactions in yeast on a genome scale. En. Nature Methods 7, 1017 (Dec. 2010).

28. Leek, J. T. et al. Tackling the widespread and critical impact of batch effects in high-throughput data. Nature Reviews Genetics 11. 733, 733+ (Oct. 2010).

29. Fisher, R. A. XV.The Correlation between Relatives on the Supposition of Mendelian Inheritance. Transactions of the Royal Society of Edinburgh 52, 399433 (1919).

30. Marchini, J., Cardon, L. R., Phillips, M. S. \& Donnelly, P. The effects of human population structure on large genetic association studies. Nature Genetics 36, 512 (2004).

31. Spor, A. et al. Hierarchical bayesian modelling for Saccharomyces cerevisiae population dynamics. International Journal of Food Microbiology 142, 25-35 (2010). 
32. Heydari, J., Lawless, C., Lydall, D. A. \& Wilkinson, D. J. Bayesian hierarchical modelling for inferring genetic interactions in yeast. Journal of the Royal Statistical Society. Series C, Applied Statistics 65, 367-393 (Apr. 2016).

33. Reimherr, M. \& Nicolae, D. Estimating Variance Components in Functional Linear Models With Applications to Genetic Heritability. Journal of the American Statistical Association 111, 407-422 (Jan. 2016).

34. Ng, W. V. et al. Genome sequence of Halobacterium species NRC-1. Proceedings of the National Academy of Sciences 97, 12176-12181 (Oct. 2000).

35. Bonneau, R. et al. A Predictive Model for Transcriptional Control of Physiology in a Free Living Cell. Cell 131, 1354-1365 (Dec. 2007).

36. Brooks, A. N. et al. A system-level model for the microbial regulatory genome. Molecular Systems Biology 10, 740 (July 2014).

37. Imlay, J. A. Pathways of oxidative damage. Annual Review of Microbiology 57, 395-418 (2003).

38. Imlay, J. A. The molecular mechanisms and physiological consequences of oxidative stress: lessons from a model bacterium. Nature Reviews Microbiology 11, 443-454 (May 2013).

39. Wu, Y., Vuli, M., Keren, I. \& Lewis, K. Role of oxidative stress in persister tolerance. Antimicrobial Agents and Chemotherapy 56, 4922-4926 (Sept. 2012).

40. Tonner, P. D., Pittman, A. M. C., Gulli, J. G., Sharma, K. \& Schmid, A. K. A Regulatory Hierarchy Controls the Dynamic Transcriptional Response to Extreme Oxidative Stress in Archaea. PLoS Genet 11, e1004912 (Jan. 2015).

41. Sharma, K., Gillum, N., Boyd, J. L. \& Schmid, A. The RosR transcription factor is required for gene expression dynamics in response to extreme oxidative stress in a hypersaline-adapted archaeon. BMC genomics 13, 351 (2012).

42. Kaur, A. et al. Coordination of frontline defense mechanisms under severe oxidative stress. Molecular Systems Biology 6, 393 (July 2010).

43. Soppa, J. From genomes to function: haloarchaea as model organisms. Microbiology 152, 585-590 (Mar. 2006).

44. Halstead, F. D. et al. The Antibacterial Activity of Acetic Acid against Biofilm-Producing Pathogens of Relevance to Burns Patients. PLoS ONE 10, e0136190 (2015).

45. Mira, N. P. \& Teixeira, M. C. Microbial mechanisms of tolerance to weak acids: an overview Google-Books-ID: jC9RBQAAQBAJ (Frontiers E-books, Nov. 2014).

46. Zakrzewska, A. et al. Genome-wide analysis of yeast stress survival and tolerance acquisition to analyze the central trade-off between growth rate and cellular robustness. Molecular Biology of the Cell 22, 4435-4446 (Nov. 2011). 
47. Altnta, A., Martini, J., Mortensen, U. H. \& Workman, C. T. Quantification of oxidative stress phenotypes based on high-throughput growth profiling of protein kinase and phosphatase knockouts. FEMS yeast research 16, fov101 (Feb. 2016).

48. Van Derlinden, E., Lule, I., Bernaerts, K. \& Van Impe, J. Quantifying the heterogeneous heat response of Escherichia coli under dynamic temperatures. Journal of Applied Microbiology 108, 1123-1135 (Apr. 2010).

49. Ihssen, J. \& Egli, T. Specific growth rate and not cell density controls the general stress response in Escherichia coli. Microbiology 150, 1637-1648 (June 2004).

50. Beer, K. D., Wurtmann, E. J., Pinel, N. \& Baliga, N. S. Model organisms retain an "ecological memory" of complex ecologically relevant environmental variation. Applied and Environmental Microbiology 80, 1821-1831 (Mar. 2014).

51. Ramsay, J. \& Silverman, B. W. Functional Data Analysis (Springer Series in Statistics) (Springer, 2005).

52. Gelman, A. Analysis of variance - why it is more important than ever. The Annals of Statistics 33, 1-53 (Feb. 2005).

53. Gelman, A. et al. Bayesian Data Analysis, Third Edition 3 edition (Chapman and Hall/CRC, Boca Raton, Nov. 2013).

54. Rasmussen, C. E. \& Williams, C. K. I. Gaussian Processes for Machine Learning (University Press Group Limited, Jan. 2006).

55. Carpenter, B. et al. Stan: A Probabilistic Programming Language. Journal of Statistical Software, Articles 76, $1-32(2017)$.

56. Solak, E., Murray-Smith, R., Leithead, W. E., Leith, D. J. \& Rasmussen, C. E. Derivative Observations in Gaussian Process Models of Dynamic Systems in Proceedings of the 15th International Conference on Neural Information Processing Systems (MIT Press, Cambridge, MA, USA, 2002), 1057-1064.

57. Hu, J., Coombes, K. R., Morris, J. S. \& Baggerly, K. A. The importance of experimental design in proteomic mass spectrometry experiments: some cautionary tales. Briefings in Functional Genomics $\mathcal{E}$ Proteomics 3, 322-331 (Feb. 2005).

58. Nicholson, G. \& Holmes, C. A note on statistical repeatability and study design for high-throughput assays. Statistics in Medicine 36, 790-798 (Feb. 2017).

59. Mecham, B. H., Nelson, P. S. \& Storey, J. D. Supervised normalization of microarrays. Bioinformatics 26, 1308-1315 (May 2010).

60. Leek, J. T. \& Storey, J. D. Capturing Heterogeneity in Gene Expression Studies by Surrogate Variable Analysis. PLoS Genetics 3, e161 (2007).

61. Ioannidis, J. P. A. Microarrays and molecular research: noise discovery? Lancet (London, England) 365, 454455 (Feb. 2005). 
62. Teschendorff, A. E., Zhuang, J. \& Widschwendter, M. Independent surrogate variable analysis to deconvolve confounding factors in large-scale microarray profiling studies. Bioinformatics 27, 1496-1505 (June 2011).

63. Johnson, W. E., Li, C. \& Rabinovic, A. Adjusting batch effects in microarray expression data using empirical Bayes methods. Biostatistics 8, 118-127 (Jan. 2007).

64. Delignette-Muller, M. L. \& Rosso, L. Biological variability and exposure assessment. International Journal of Food Microbiology 58, 203-212 (July 2000).

65. Koutsoumanis, K. P. \& Sofos, J. N. Effect of inoculum size on the combined temperature, pH and $\mathrm{A}_{w}$ limits for growth of Listeria monocytogenes. International Journal of Food Microbiology 104, 83-91 (Sept. 2005).

66. Ryall, B., Eydallin, G. \& Ferenci, T. Culture history and population heterogeneity as determinants of bacterial adaptation: the adaptomics of a single environmental transition. Microbiology and molecular biology reviews: MMBR 76, 597-625 (Sept. 2012).

67. Jaloustre, S., Guillier, L., Morelli, E., Nol, V. \& Delignette-Muller, M. L. Modeling of Clostridium perfringens vegetative cell inactivation in beef-in-sauce products: A meta-analysis using mixed linear models. International Journal of Food Microbiology 154, 44-51 (Mar. 2012).

68. Cao, R., Francisco-Fernndez, M. \& Quinto, E. J. A random effect multiplicative heteroscedastic model for bacterial growth. BMC Bioinformatics 11, 77 (2010).

69. Bradley, M. D., Neu, D., Bahar, F. \& Welch, R. D. Inter-laboratory evolution of a model organism and its epistatic effects on mutagenesis screens. Scientific Reports 6 (Dec. 2016).

70. Draper, J. L. et al. Fallacy of the Unique Genome: Sequence Diversity within Single Helicobacter pylori Strains. mBio 8 (Feb. 2017).

71. Peck, R. F., Dassarma, S. \& Krebs, M. P. Homologous gene knockout in the archaeon Halobacterium salinarum with ura3 as a counterselectable marker. Mol Microbiol 35, 667-76 (2000).

72. Kahm, M., Hasenbrink, G., Lichtenberg-Frat, H., Ludwig, J. \& Kschischo, M. grofit: Fitting Biological Growth Curves with R. Journal of Statistical Software, Articles 33, 1-21 (2010).

73. Jones, E., Oliphant, T., Peterson, P., et al. SciPy: Open source scientific tools for Python 2001.

74. Kaufman, C. G. \& Sain, S. R. Bayesian functional ANOVA modeling using Gaussian process prior distributions. Bayesian Analysis 5, 123-149 (Mar. 2010).

75. Brooks, S. P. \& Gelman, A. General Methods for Monitoring Convergence of Iterative Simulations. Journal of Computational and Graphical Statistics 7, 434-455 (1998). 
bioRxiv preprint doi: https://doi.org/10.1101/793174; this version posted October 4,2019 . The copyright holder for this preprint (which was not certified by peer review) is the author/funder, who has granted bioRxiv a license to display the preprint in perpetuity. It is made available under aCC-BY-NC-ND 4.0 International license.

\section{${ }_{501} \quad 6 \quad$ Supplementary Material}



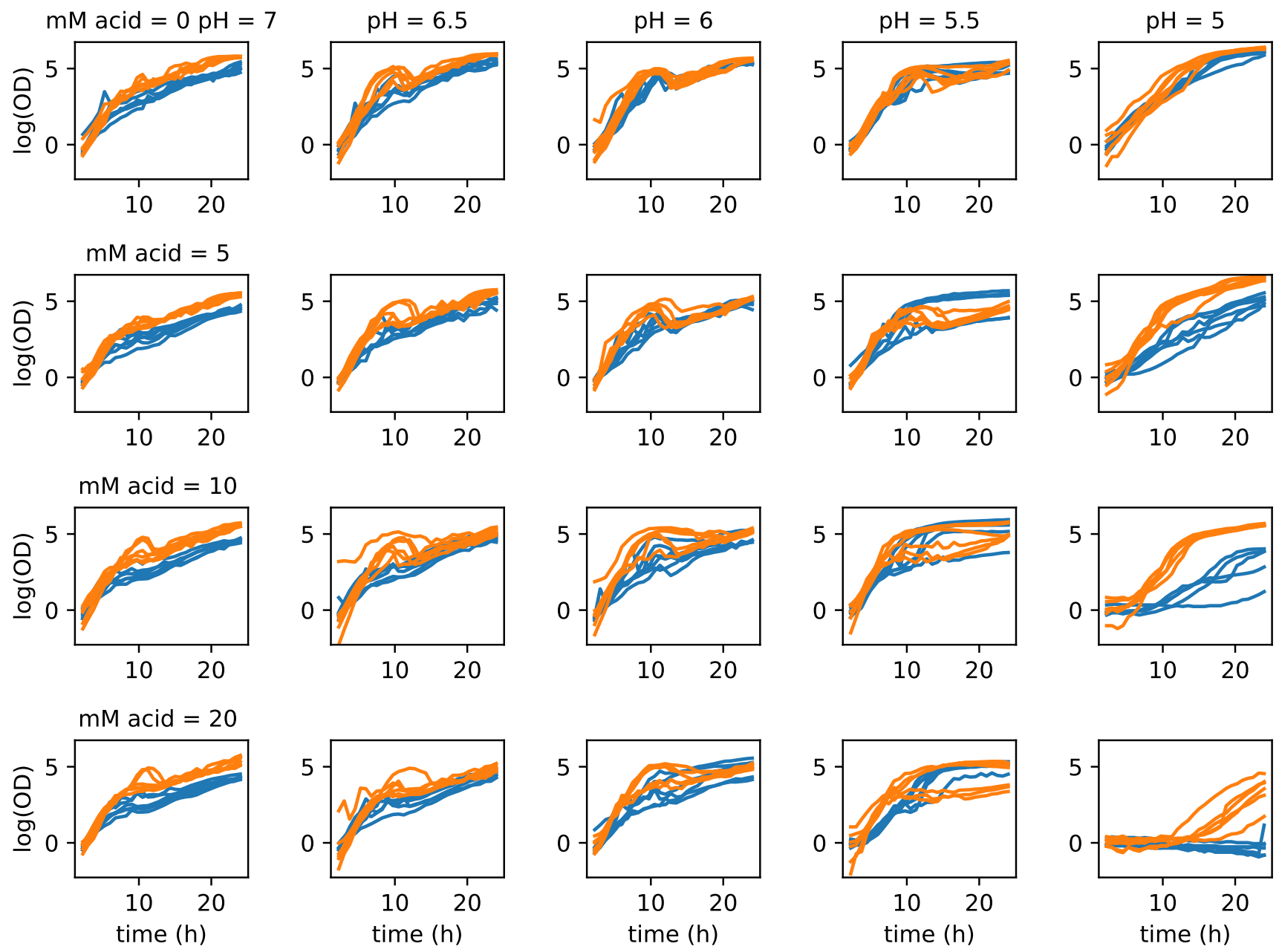

Figure S1: $\boldsymbol{P}$. aeruginosa growth under benzoate and pH gradient. Growth of $P$. aeruginosa strain PA01 under gradient of $\mathrm{pH}(7-5)$ and benzoate $(0-20)$. Colors represent different batches. 

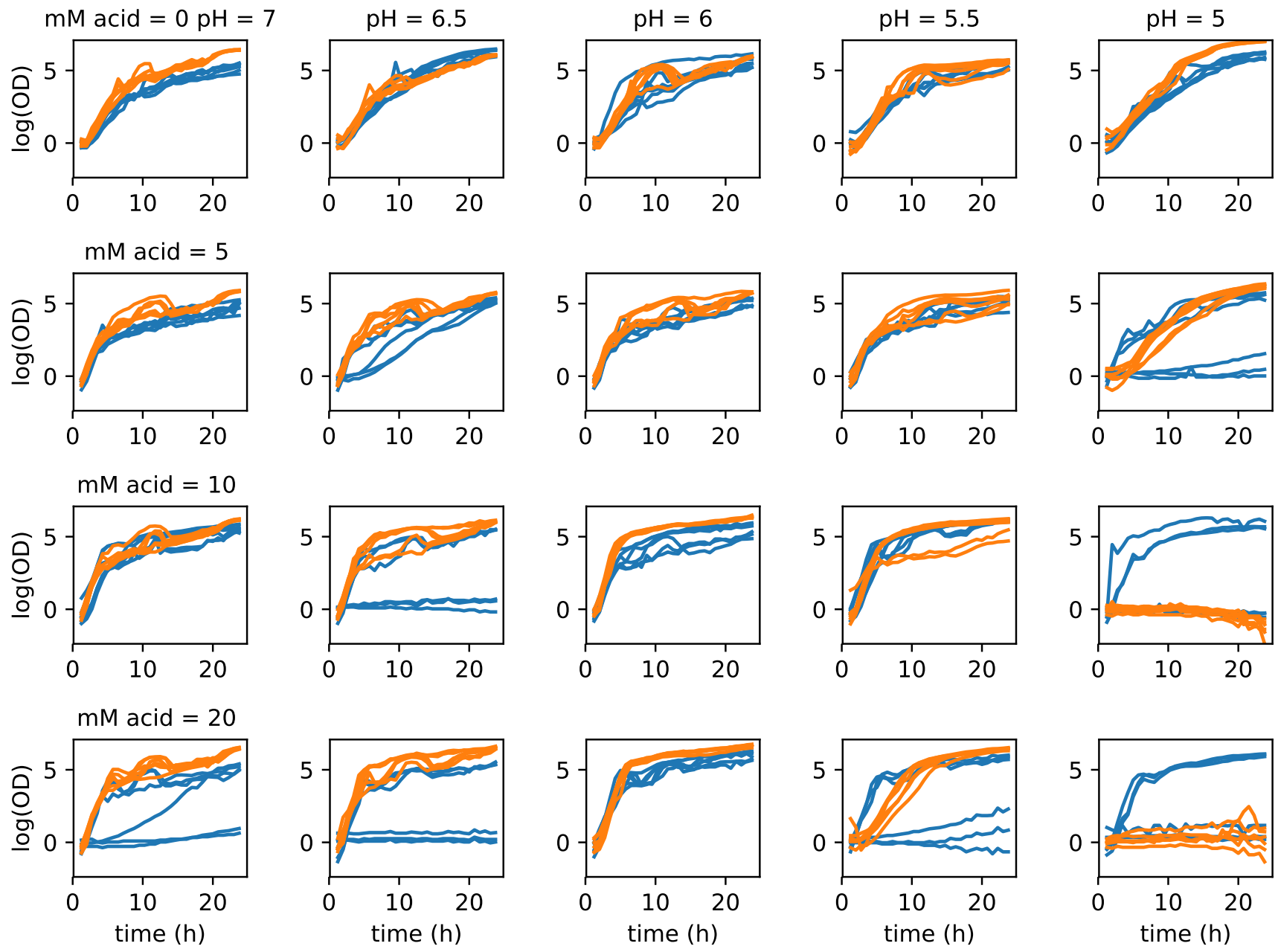

Figure S2: $\boldsymbol{P}$. aeruginosa growth under malic acid and pH gradient. Growth of $P$. aeruginosa strain PA01 under gradient of $\mathrm{pH}(7-5)$ and malic acid $(0-20)$. Colors represent different batches.

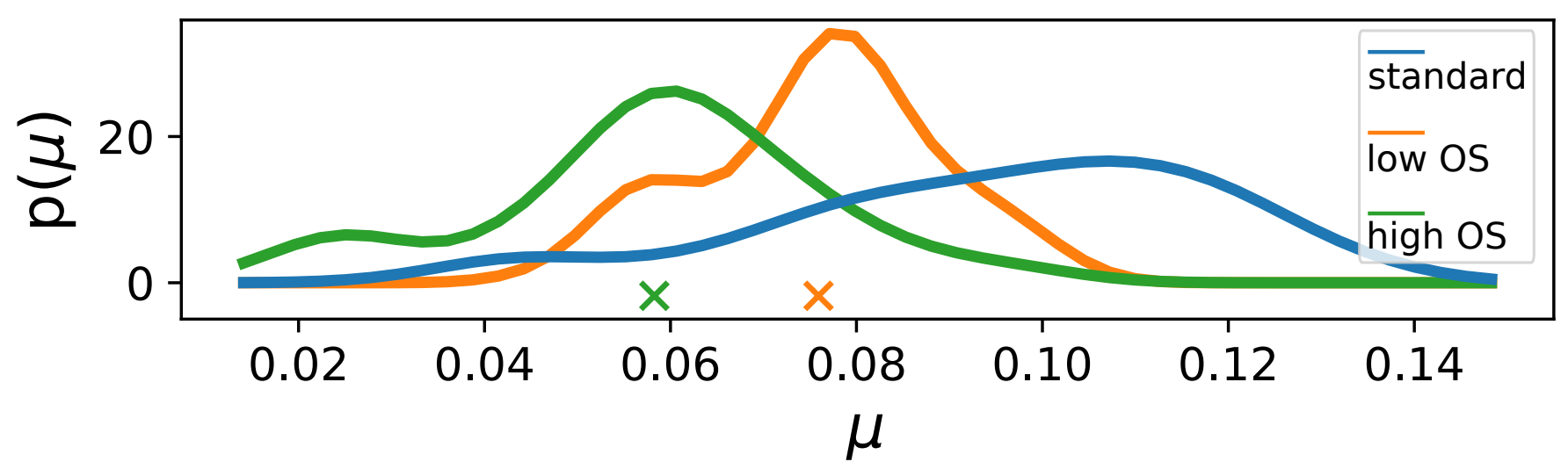

Figure S3: KDE of $\mu_{\max }$ for $\boldsymbol{H}$. salinarum growth across batches. Crosses indicate significant difference between $\mu_{\max }$ standard conditions and each OS level (one-sided t-test, $p<0.05$ ) 


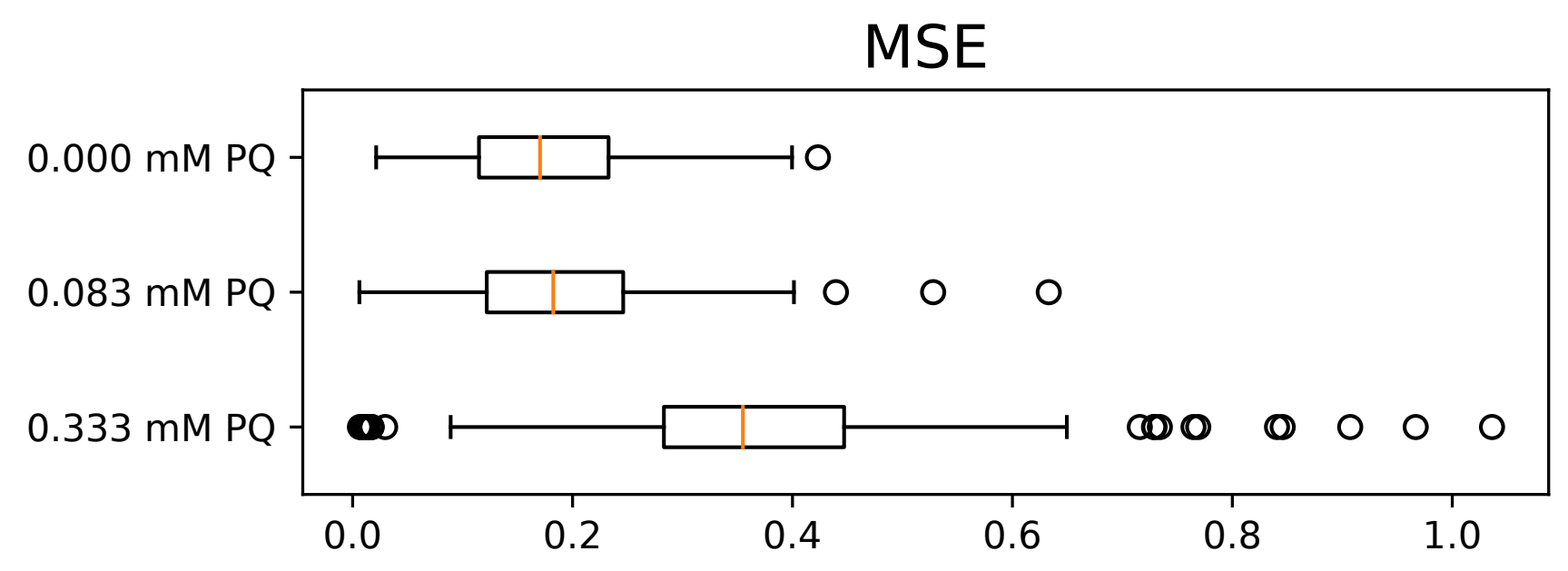

Figure S4: Error in parametric growth models. Distribution of error (MSE) for each condition when fit with a logistic growth curve. The box show shows the inter-quartile range, red line is the median, whiskers show the 1.5 inter-quartile range, and the individual points are outliers.
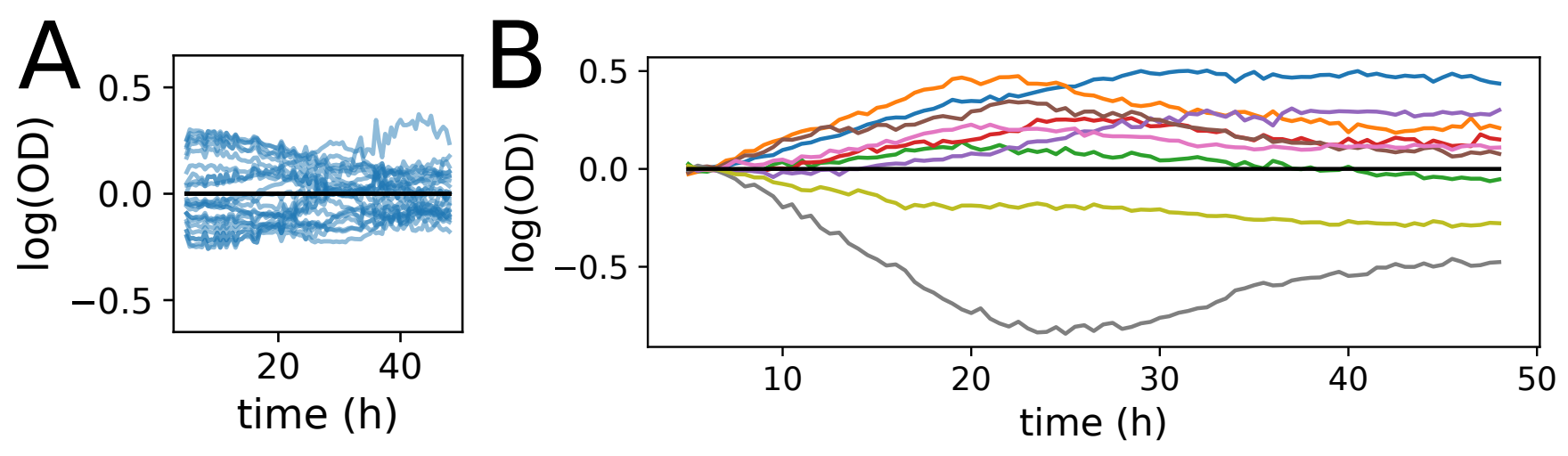

Figure S5: Residual structure of microbial growth data across batches. (A) Individual replicate curve residuals around the mean of the respective batch. Only standard conditions are shown. (B) Residual of the mean behavior for each batch around the global mean (standard condition only). 


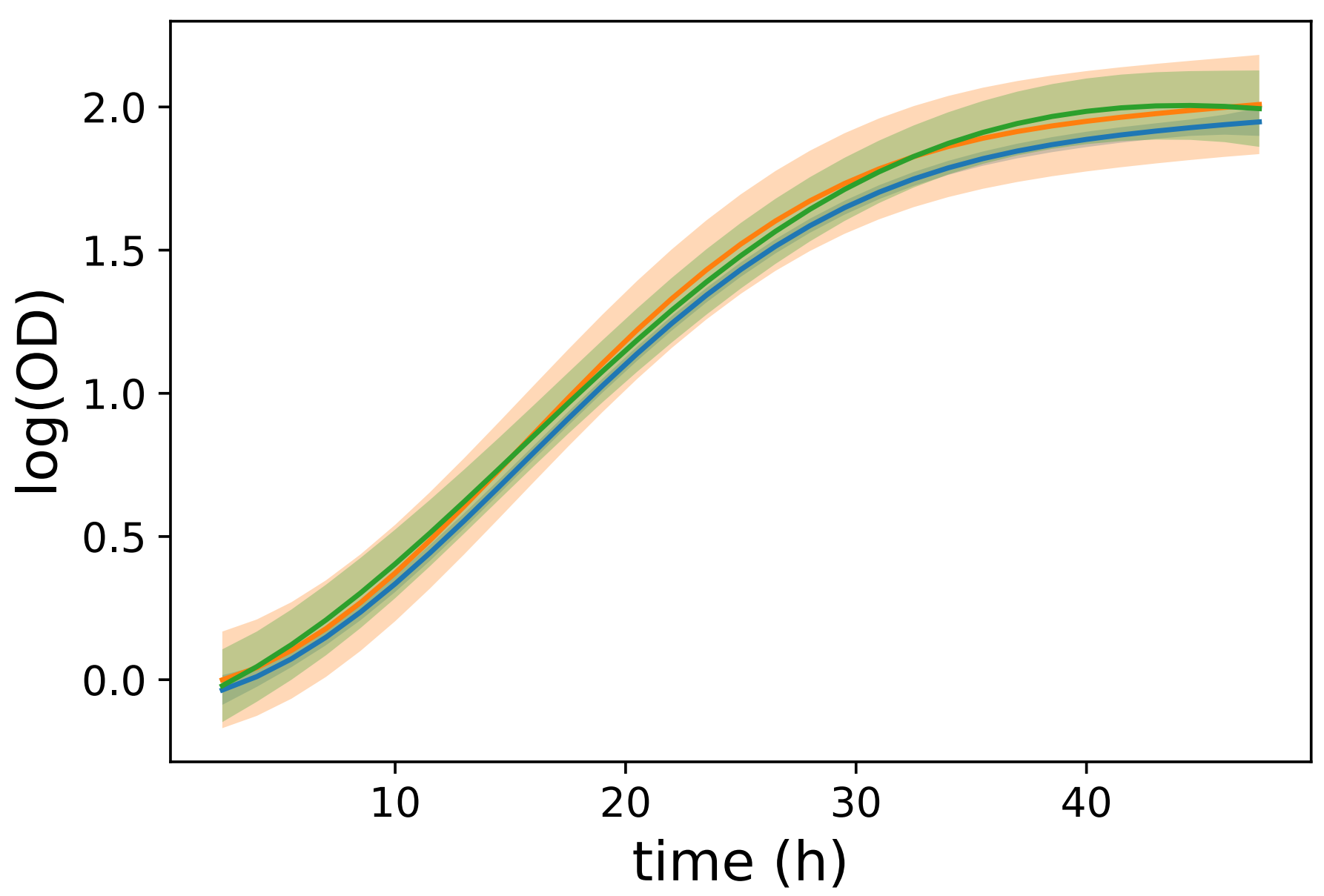

Figure S6: Posterior comparison of $\mu(t)$ for $H$. salinarum growth across batches. Posterior interval of $\mu(x)$ for H. salinarum standard growth.

A

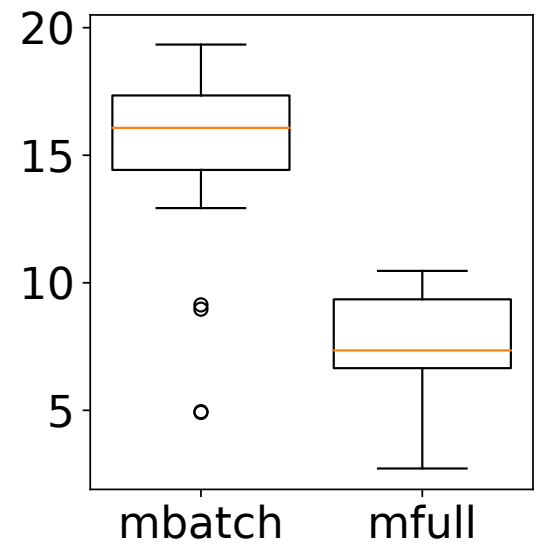

B

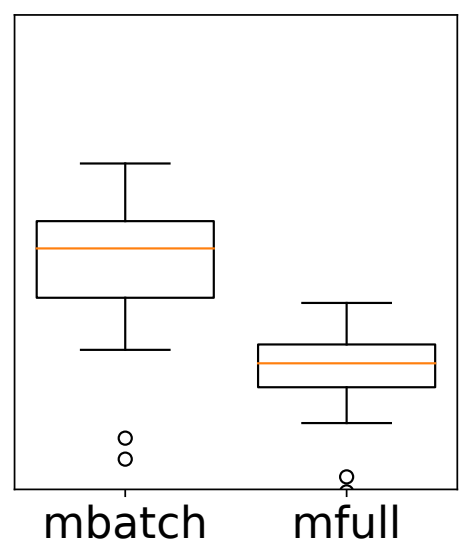

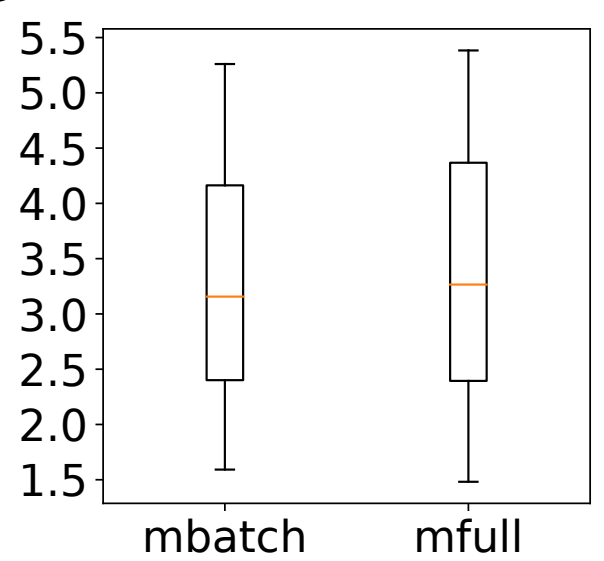

Figure S7: Posterior variance of function estimates under different models. Each plot shows the posterior variance of a function at each time point under each of $\mathrm{M}_{\text {batch }}$ and $\mathrm{M}_{\text {full }}$ versus $\mathrm{M}_{\text {null }}$. (A) $\delta(x)$ estimated for $H$. salinarum growth under low (left) and high (right) OS. (B) $(\alpha \beta)_{p, m}(t)$ at $\mathrm{pH}=5, \mathrm{mM}$ malic acid $=10$. 